\title{
Evolution des représentations et des systèmes paysagers en Transylvanie (L'exemple du département de BistriŢa-Năsăud)
}

Evolution of the representations and the landscaped systems in Transylvania

(The example of the county of BistriŢa-Năsăud)

Claire Coumeff-Toader et Dominique Harmand

\section{OpenEdition}

Édition électronique

URL : http://journals.openedition.org/rge/1856

DOI : $10.4000 /$ rge.1856

ISSN : 2108-6478

Éditeur

Association des géographes de l'Est

Édition imprimée

Date de publication : 1 janvier 2009

ISSN : 0035-3213

\section{Référence électronique}

Claire Coumeff-Toader et Dominique Harmand, «Evolution des représentations et des systèmes paysagers en Transylvanie (L'exemple du département de Bistrița-Năsăud) », Revue Géographique de l'Est [En ligne], vol. 49 / 1 | 2009, mis en ligne le 16 juillet 2010, consulté le 08 septembre 2020. URL : http://journals.openedition.org/rge/1856 ; DOI : https://doi.org/10.4000/rge.1856

Ce document a été généré automatiquement le 8 septembre 2020

Tous droits réservés 


\title{
Evolution des représentations et des systèmes paysagers en Transylvanie (L'exemple du département de BistriŢa-Năsăud)
}

\author{
Evolution of the representations and the landscaped systems in Transylvania \\ (The example of the county of BistriŢa-Năsăud)
}

Claire Coumeff-Toader et Dominique Harmand

La Transylvanie historique (fig. 1, 2), une des provinces roumaines, appartient aux espaces «d'entre-deux» (Rey, 1998; Rey et Groza, 2008), ces régions d'Europe centrale et orientale caractérisées par la pluri-appartenance et la multi-temporalité. En témoigne son caractère pluriethnique, quoique très amenuisé depuis 1945: des populations majoritairement roumaines, mais aussi magyares, allemandes («Saxons»), roms et juives s'y côtoient. Saxons et Magyars ont conscience d'appartenir à des confins, un espace culturel trans-étatique constitué sur une base ethnolinguistique. La province a connu les vicissitudes des dominations politiques et des influences culturelles: sous autorité autrichienne à partir de 1691, elle passa sous administration hongroise en 1867, puis fut intégrée à la Grande Roumanie de l'Entre-deux-guerres; sa partie nord fut occupée par la Hongrie pendant la Seconde guerre mondiale; enfin, elle subit le régime communiste (modèle soviétique) jusqu'en 1989, année qui ouvrit la période récente d'intégration progressive à l'Union Européenne. Les paysages ruraux transylvains actuels portent les marques de cette histoire, les empreintes juxtaposées ou superposées des systèmes qui s'y sont succédés.

Une analyse des dynamiques paysagères sur plus d'un siècle montre que le jeu de l' entre-deuxmet en valeur leurs ressorts généraux. Le terrain choisi est le département de BistriŢa-Năsăud (fig. 3). Son intérêt est, d'une part, de concentrer des types de paysages représentatifs de la Transylvanie (plateau au sud, collines, moyenne montagne au nord et à l'est) et d'offrir un échantillon d'espaces ruraux peuplés de populations roumaines, magyares et même saxonnes, touchés inégalement par la 
collectivisation (Rey et al., 2000), et d'autre part, d'illustrer l'éventail de représentations paysagères multipliées et détruites, importées et exportées par le jeu de l'entre-deux. Les systèmes paysagers de trois villages du département ont été particulièrement étudiés durant la période austro-hongroise et l'Entre-deux-guerres, la période communiste et l'époque actuelle. Chaque rupture permet de voir comment les fluctuations historiques provoquent une défiguration/reconfiguration des paysages plus ou moins profonde. Le géographe peut alors proposer une interprétation de leur évolution en termes de généalogies de paysages que la situation d'entre-deux rend plus nettement sensibles.

Fig. 1 : localisation du département de Bistrita-Nasaud et des communes étudiées.

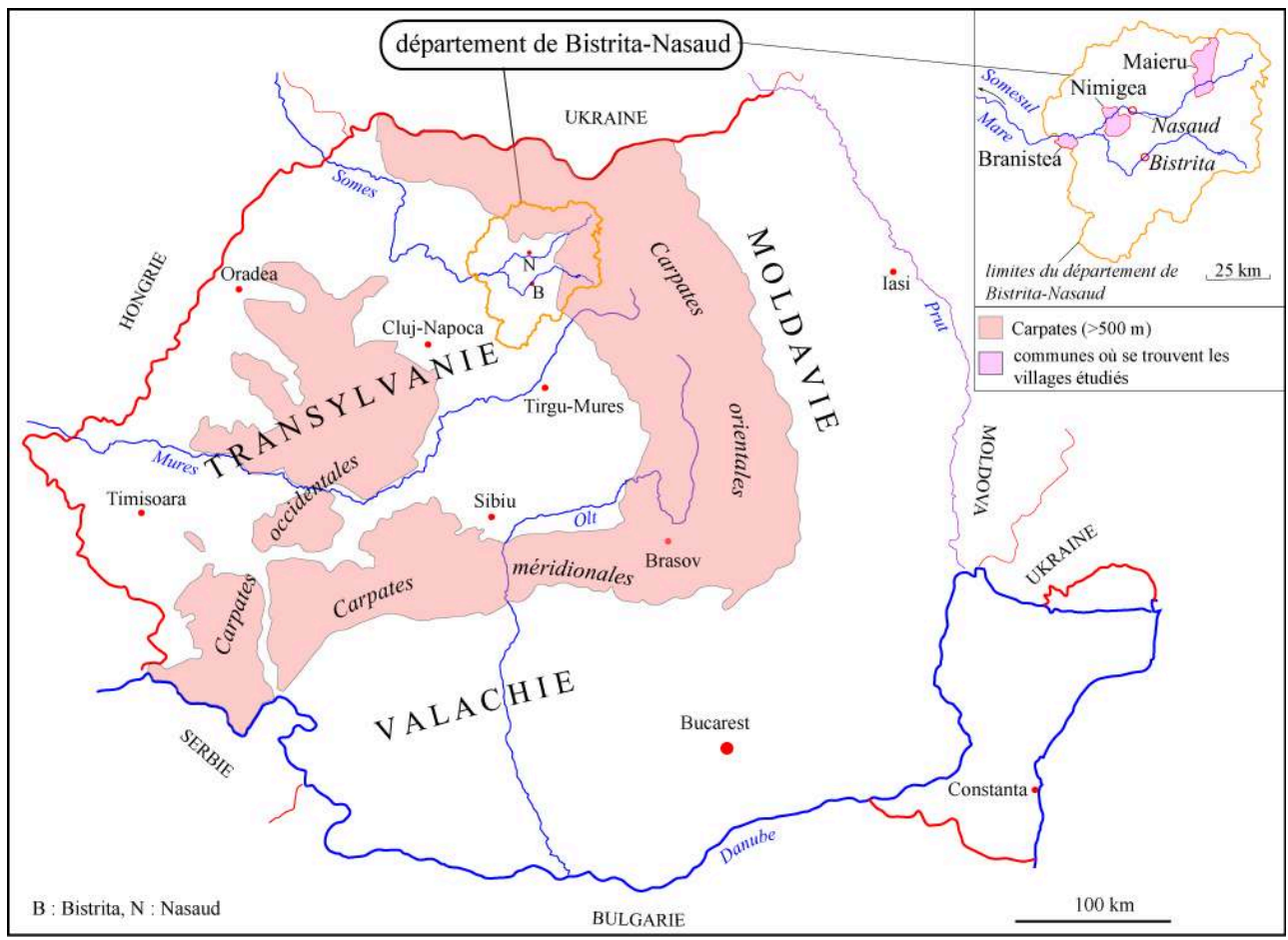


Fig. 2 : les confins militaires de l'Empire d'Autriche à la fin du XVIIle siècle (d'après Nouzille, 1991, et d'autres auteurs)

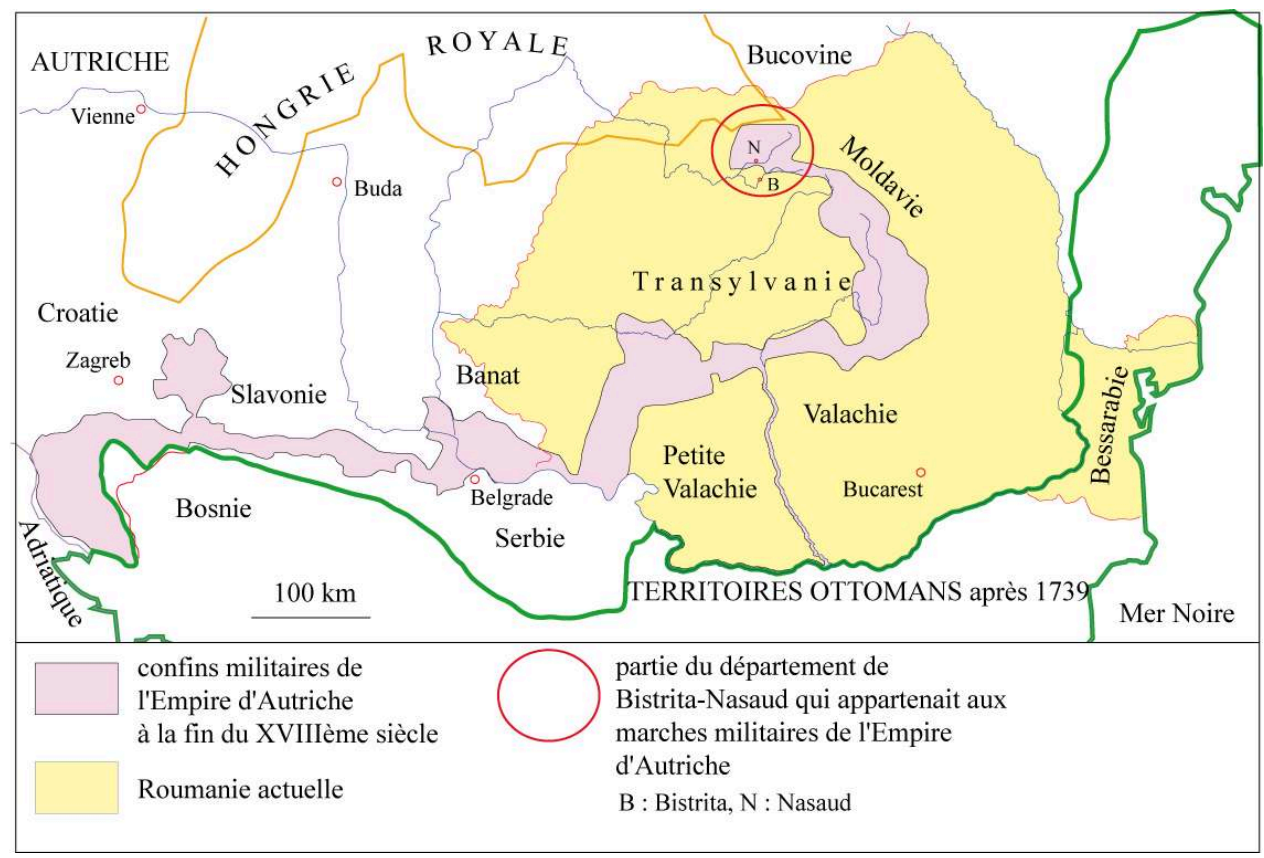

\section{1- Principes d'interprétation des dynamiques paysagères en situation d'entre-deux}

L'ensemble de l'étude est fondé sur le concept d'entre-deux. Le premier principe de l'analyse est d'appréhender le paysage dans sa globalité, pour mieux en comprendre l'évolution. Si l'on tient que la réalité du paysage naît de sa dualité, "ni seulement objectif, ni seulement subjectif, mais résultant de l'entrecroisement d'éléments et de relations de l'un et de l'autre mondes" (Rougerie et Beloutchachvili, 1991), que sa réalité émerge d'un va-et-vient en spirale (Berque, 1995) entre paysage-empreinte et paysage-matrice, alors, les dynamiques paysagères doivent être étudiées selon cette double et indissoluble approche. Celle-ci nécessite un découpage conceptuel échappant au paradigme nature/société. En découle un second principe d'interprétation. Prévélakis regrette que la dialectique circulation/iconographie proposée par Gottmann (in Prévélakis, 2001) n'ait pas davantage suscité l'intérêt des géographes : circulation d'éléments physiques et humains face à laquelle une société se protège grâce à son iconographie, un réseau de symboles construit autour de la religion, du passé politique et de l'organisation sociale. Bruneau (2000) a pointé le «rapport nécessaire de l'iconographie à un support géographique »: une topographie, une végétation ou une architecture singulières sont mobilisées par une société pour forger un signe paysager associé à son iconographie. La grille d'analyse de Gottmann permet donc d'envisager la complexité tant des empreintes que des représentations paysagères en Transylvanie.

Ici, les flux et reflux géopolitiques et culturels ont tantôt favorisé, tantôt freiné ou réorienté la circulation, face à laquelle les sociétés, à l'échelle régionale ou locale, ont tenté de résister et d'assurer la survie de leur système paysager grâce à leur iconographie. En même temps, les paysages de Transylvanie ont été mis en images, en récits, et même en scène. Les populations paysannes, particulièrement dans le 
département de Bistrị̧a-Năsăud, font preuve d'un sens paysager qui s'exprime dans les arts populaires (motifs floraux récurrents des vêtements, des icônes sur verre, des chants ...) et dans l'entretien du jardinet clos de chaque maison villageoise, à la fonction décorative et religieuse (Coumeff-Toader, 2008). Les paysages ruraux transylvains ont aussi nourri la littérature: Rebreanu (1885-1944), grand écrivain roumain natif du département de BistriŢa-Năsăud, y situe l'action de son roman Ion et appelle la vallée où se blottit le village de son enfance «le nid des rêves" (Rebreanu, 1980, 1984). Surtout, les représentations relatives aux paysages transylvains prennent appui sur des caractéristiques topographiques et végétales singulières: une vaste cuvette où alternent collines, plateaux et larges vallées, comme celle du Someş Mare, cernée par les Carpates, un cadre montagneux où la forêt domine et dont les lignes de crête et les cols coïncident pratiquement partout avec les limites historiques de la province. Elle a suscité dans l'imaginaire collectif la figure paysagère nationale de la forteresse (von Hirschhausen, 2003). De cette matrice commune divergent des représentations inversées, celles des Magyars et Saxons, d'un côté, celles des Roumains, de l'autre, à cause du jeu de l'entre-deux vécu entre 1867 et 1945 (Walter, 2004). Pour les uns, des événements traumatisants (traité de Trianon pour les Hongrois, Seconde guerre mondiale pour les Saxons) ont contribué à connoter de valeurs intemporelles les paysages mythiques de ces confins perdus (de Oliveira, 2005; de Trégomain, 2005) (photo. 1). Pour les autres, la construction de l'État-Nation s'est accompagnée d'une idéologisation des paysages ruraux transylvains qui justifie a posteriori la légitimité des frontières nationales; le paysage se dédouble en deux figures: l'espace de la transhumance (les estives) et le village, célébrés par le philosophe roumain transylvain Blaga (1895-1961) pour qui l'» inconscient spirituel » collectif roumain est modelé par un horizon spatio-temporel investi de valeurs religieuses, une "matrice stylistique ", (Blaga, 1937, 1991) à l'image du plai [pâturage d'altitude] de l'est du département (photo. 2). Le style né de cette matrice se retrouverait dans le paysage villageois (Durandin, 1995). Cette représentation a pu dériver vers un nationalisme exclusif (Mungiu-Pippidi, 2004, Boia, 2003) qui entretient la représentation de paysages ruraux immuables. Le régime communiste manipula ces figures paysagères pour mieux masquer une vision totalitaire impliquant une entreprise de destruction et de recomposition du paysage rural. Celle-ci toucha les zones occidentales et méridionales du département où des coopératives agricoles de production (CAP) furent imposées aux populations.Aujourd'hui, trois imaginaires portés en force par les populations d'Europe occidentale et d'Amérique du Nord se dégagent : le cliché d'un paysage " authentique ", proche de celui du paysage immobile des Roumains, parfois confondu avec le paysage " ethnique » des villages fortifiés saxons, et le paysage "fantastique ", le plus original et le plus décalé par rapport aux représentations locales. Car les croyances roumaines dans des «revenants» (strigoi) - une possible métaphorisation de la relation ambivalente des sociétés d'Europe centrale et orientale à la domination turque (Montaclair, 1997) - ne sont jamais associées au cliché paysager occidental : le château isolé au sommet d'une montagne couverte d'une épaisse forêt et coupée de gorges profondes (récits d'Alexandre Dumas (1849), de Jules Verne (1892) et bien sûr de Bram Stoker (1897) qui inventa le personnage de Dracula le vampire). Le travail du géographe tend donc, non seulement à déconstruire cette face subjective du paysage pour la confronter à l'analyse objective de systèmes paysagers, mais aussi à prendre en compte ces représentations paysagères, particulièrement la puissance évocatrice du plai, le «nid» des vallées transylvaines, l'image de la forteresse et l'ambiguïté de la vision 
fantastique comme autant de matrices possibles, parties prenantes de l'iconographie des sociétés. L'objectif est de proposer une reconstruction des dynamiques paysagères fondée sur ces principes.

\section{Photographie 1 : La tour de guet d'un ancien village saxon, Dumitra, août 2008, C. Coumeff-Toader}

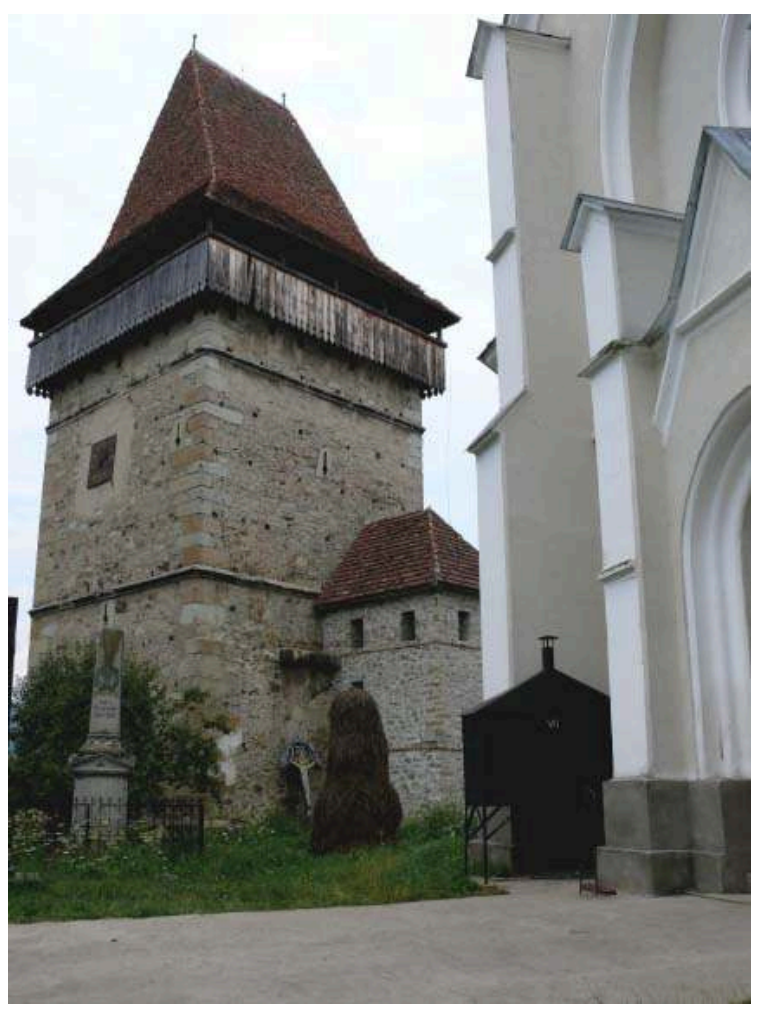

Le village était peuplé de descendants de paysans-soldats venus d'Allemagne rhénane au Moyen-Âge pour défendre la frontière; le monument aux morts porte des noms allemands écrits en lettres gothiques. La tour de guet du XVe siècle de l'ancienne église allemande côtoie l'actuelle église orthodoxe sur une hauteur surplombant le village. 


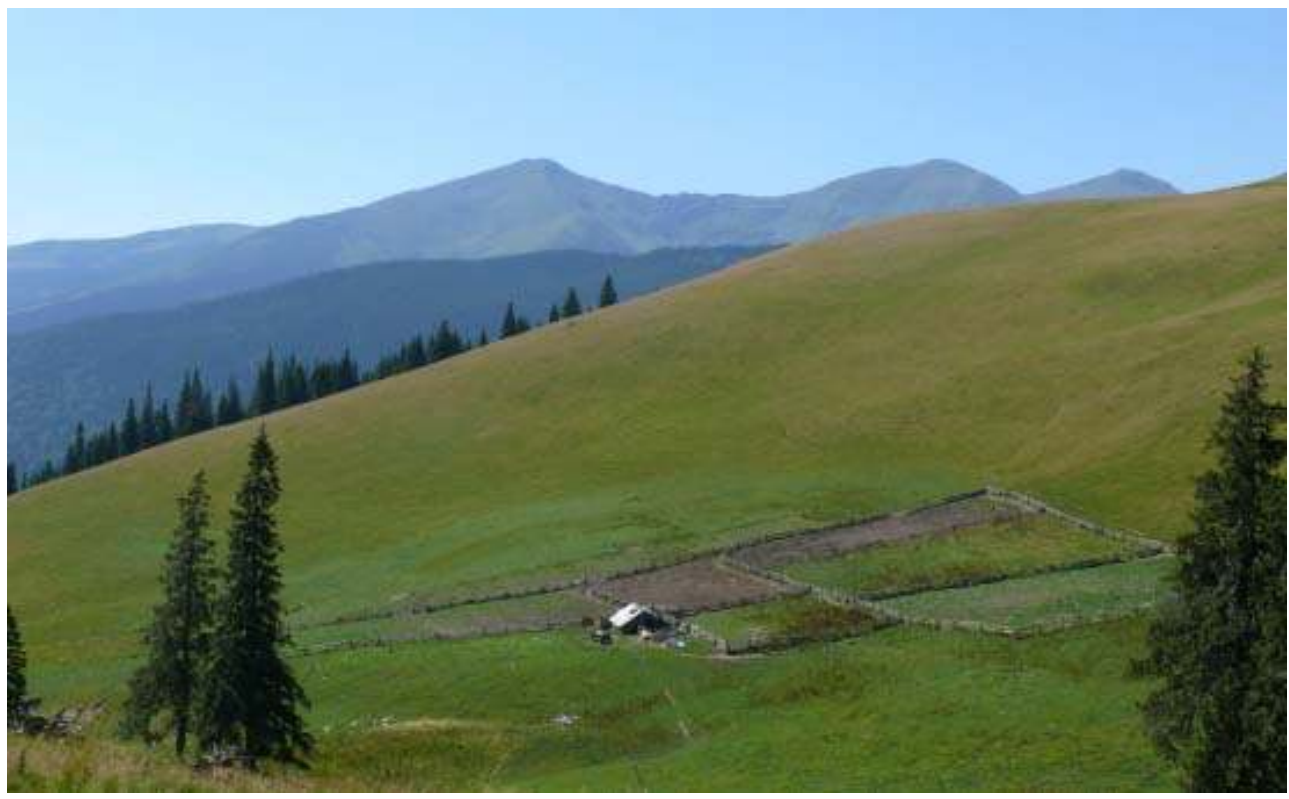

Pour Blaga, l'expérience de la transhumance serait la source de l'intériorisation par les Roumains du paysage du plai, I' » espace mioritique ». Au premier et second plans, un parc pour rassembler les animaux la nuit et les estives. À l'arrière plan, l'étage forestier, qui correspond aux représentations occidentales de l'épaisse forêt des redoutables montagnes de Transylvanie.

L'analyse des systèmes paysagers a été menée à l'échelle locale, en 2006-2007, à partir d'enquêtes sur le terrain (observations et entretiens) et de consultations d'archives (cartes, textes, photographies). Les cas de trois finages de la haute et moyenne vallée du Someş - un des deux grands axes fluviaux du département de Bistrị̧a-Năsăud - Maieru en moyenne montagne, Cireşoaia dans une zone de collines, Floreşti dans la plaine alluviale (fig. 3), ont été choisis pour leur représentativité : type de relief, données démographiques, ethniques et religieuses disponibles, histoire. La grille d'analyse reprend les principes de fonctionnement des géosystèmes (Bertrand, 2002), de façon souple, en y intégrant les flux pour mieux mettre à jour les effets de la dialectique circulation/iconographie. L'appréhension du système paysager, à chaque grand moment historique, repose sur la combinaison d'unités paysagères définies par leurs composantes visibles et par les pratiques des acteurs. On considère qu'à l'échelle locale, l'iconographie d'une société associe les récits fondateurs de la mémoire collective, les croyances religieuses, le type d'organisation sociale et les coutumes d'usage des composantes naturelles du finage. Les études locales ont permis de vérifier comment l'entre-deux a amplifié les occasions de ruptures paysagères et les tentatives parfois désespérées de continuité des populations accrochées à leur iconographie. 
Fig. 3 : Localisation des villages étudiés dans le département de Bistrita-Nasaud

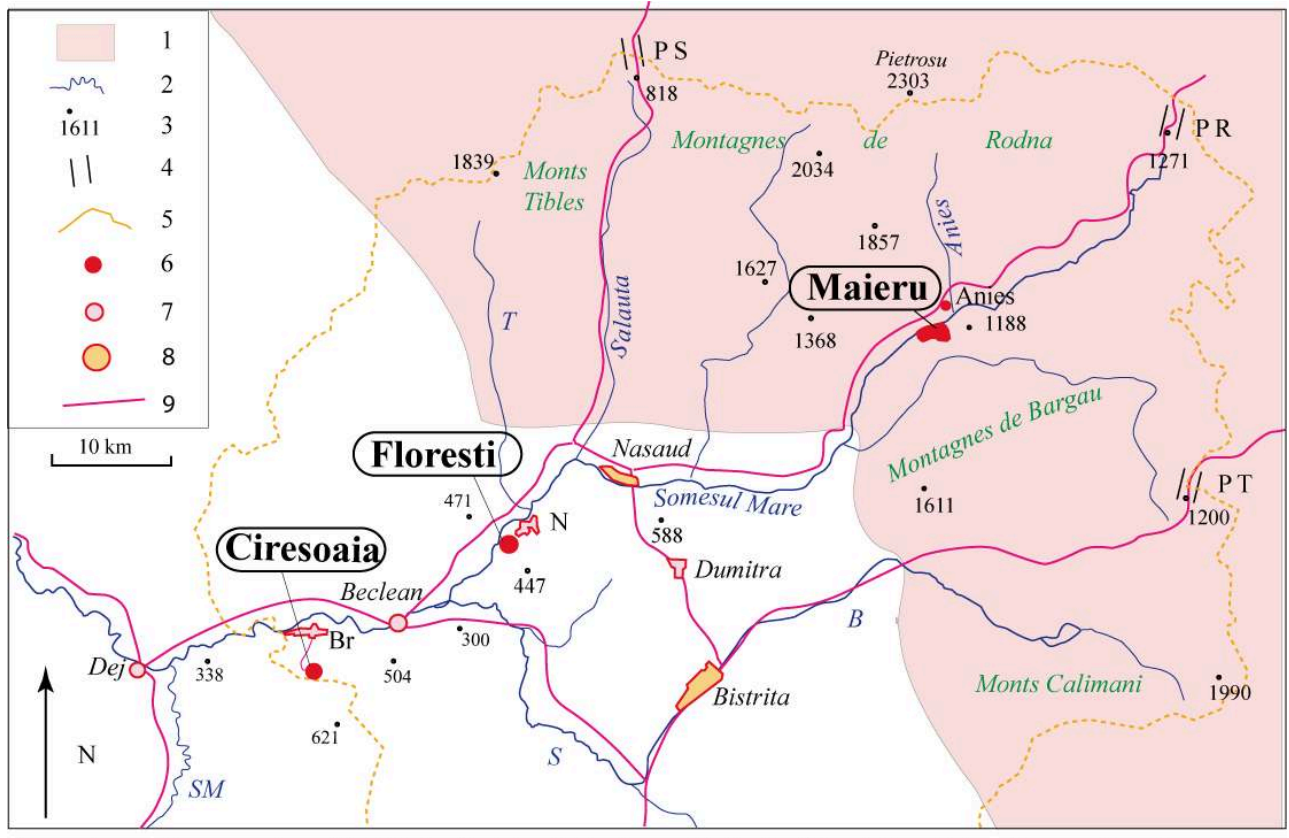

1 : montagnes, 2 : cours d'eau (B : Bistrita, $\mathrm{S}:$ Sieu, SM : Somesul Mic, T : Tibles), 3 : points cotés en mètres, 4 : principaux cols (P R : Pasul Rotunda, P S : Pasul Setref, P T : Pasul Tihuta ou Col de Bargau), 5 : limites du département de Bistrita-Nasaud, 6 : villages étudiés, $7:$ chefs-lieux de communes (B : Branicea, $\mathrm{N}:$ Nimigea), $8:$ principales villes, 9 : principales routes

\section{2 - Maieru, un système paysager de moyenne montagne ou la fausse continuité}

Gros village blotti au fond d'une dépression intramontagnarde au pied des Montagnes de Rodna, riches en gisements miniers, traversé d'est en ouest par le Someş Mare, Maieru (fig. 1, 3, 4) dispose d'un vaste finage (12875 ha) orienté nord-sud et étagé de 500 à $2000 \mathrm{~m}$, au nord-est du département. Ce cas invite à interroger cette impression de paysage immobile que les montagnes de Transylvanie évoquent chez les Roumains et les Occidentaux. Trois éléments composent son iconographie. Attesté dès le XVe siècle, il fut d'abord un village de bergers, de mineurs et de forestiers roumains. Ces activités nécessitaient des déplacements sur de longues distances, durant de longues périodes (estivage et remues) : les mentalités dès le XVIIIe siècle avaient intégré un "savoirmigrer " (von Hirschhausen, 2003). Ensuite, la mémoire collective reste marquée par l'histoire des confins militaires de l'Empire d'Autriche (figure 2) : dans quarante-quatre "communes de frontières» de l'Est du département, les hommes de religion grécocatholique furent sollicités pour surveiller les cols des Carpates de 1763 à 1851 ; pour encourager ces gardes-frontières, les autorités autrichiennes les comparèrent aux fantassins daco-roumains et fondèrent des écoles de langue roumaine, contribuant au développement de la conscience nationale roumaine (Nouzille, 1993) ; Gusti et al. (1938) insistent sur la psychologie propre aux habitants, la fierté d'un " passé héroïque » qui s'exprime par un mélange d'esprit d'indépendance et de discipline et par le besoin de reconnaissance $\mathrm{du}$ particularisme local, un sentiment encore notable chez les personnes interrogées lors des enquêtes. Entre 1867 et 1918, les habitants de la région résistèrent fortement à la magyarisation. Enfin, entre dans cette iconographie le 
système agro-pastoral propre aux populations montagnardes (von Hirschhausen, 2003) ; la mise-en-valeur des ressources des bas du finage (cultures et prés, en petites propriétés familiales) et des hauts (forêts et estives, dont la majeure partie appartenait à la collectivité) assurait l'autosubsistance et une solidarité interne assez solide pour résister à d'autres logiques socio-économiques. Cette iconographie est remarquable par sa richesse, sa cohérence et l'intégration d'une nécessaire mobilité entre différents espaces de vie. Le système paysager s'organise en trois grands étages.

Fig. 4 : évolution du géosystème de Maieru entre la fin du XIXe siècle et le début du XXle siècle

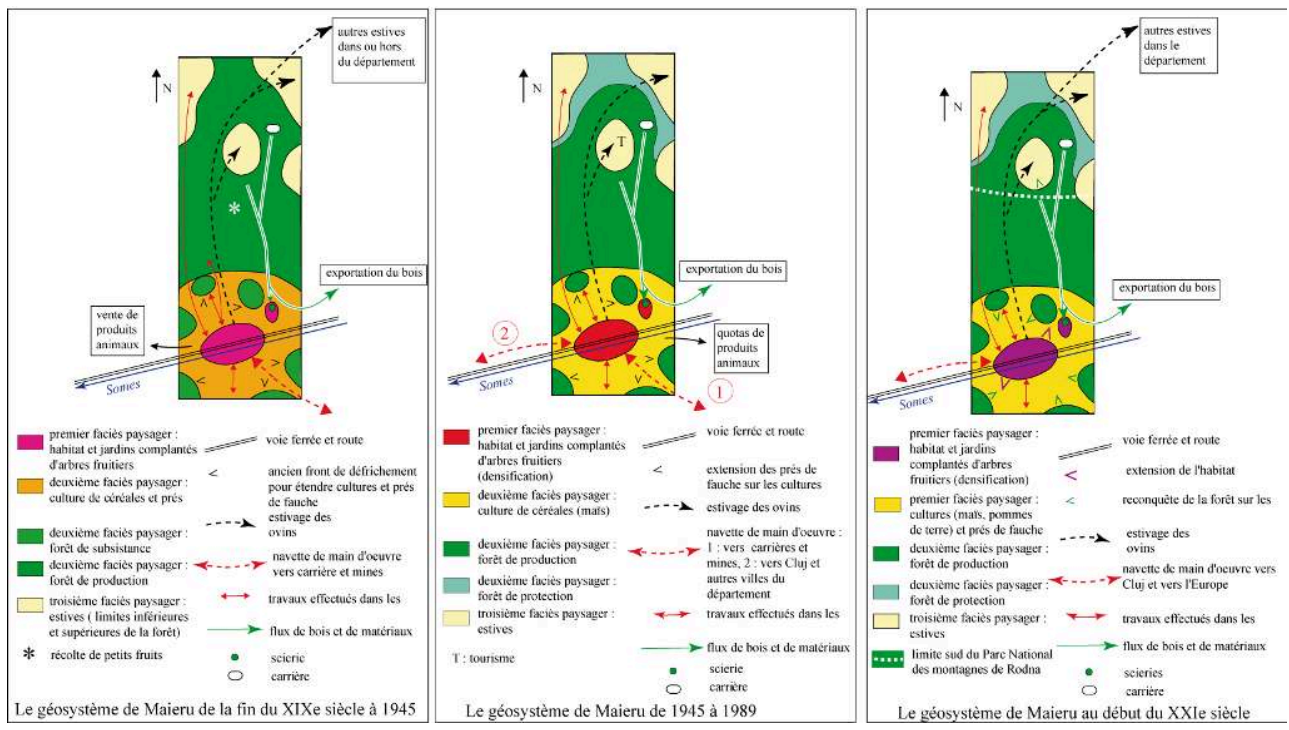

Du début du XXe siècle au lendemain de la Seconde guerre mondiale, le géosystème dut répondre aux besoins d'une population croissante. Les paysages de Maieru sont un des modèles des descriptions de Ion qui permettent de reconstituer l'aspect du premier étage paysager avant 1914 (Rebreanu, 1980): un habitat non jointif de maisons paysannes en bois, ceintes d'un balcon couvert par leur toit de chaume, entourées d'un jardin potager complanté d'arbres fruitiers (l'intravilan). Pendant l'Entre-deux-guerres, les bardeaux remplacèrent le chaume. Le village est dominé par trois măguri (monts) aux pentes douces culminant à $1000 \mathrm{~m}$ environ, que Rebreanu décrit comme « un corps (...) taillé en milliers de morceaux ", allusion au parcellaire lanièré. Les versants étaient largement cultivés : l'auteur cite blé, maïs et avoine, les témoignages oraux y ajoutent l'orge et confirment la dominante des teintes jaunes, côtoyant le vert des prés de fauche, et, dans le fond de la vallée, des parcelles de luzerne et de trèfle. En effet, il fallut nourrir une population et des troupeaux de plus en plus nombreux des années 1920 aux années 1930 (16 000 brebis et presque 9000 bovins en 1945, d'après les témoins interrogés). Pour élargir les prés de fauche et les surfaces labourées, le couvert forestier des collines recula et on aménagea des terrasses; cette forêt de subsistance très sollicitée à la fin du XIXe siècle pour la construction des maisons et dans les années 1920 pour le bois de chauffe devint de plus en plus discontinue. Les deux autres étages paysagers - forêts et estives - constituaient une ressource saisonnière: bois de construction, petits fruits des bois, pâturages. Dès l'Entre-deux-guerres, la dynamique démographique fait de Maieru un " géosystème plein » et le simple jeu des flux internes ne permet pas d'en expliquer le fonctionnement. 
En effet, celui-ci doit être intégré à un système plus vaste selon une circulation régionale (axes est-ouest) propre à la Transylvanie du Nord, que le rattachement officiel à la Roumanie en 1921 n'a pas fondamentalement perturbée. Dès la fin du XIXe siècle, d'autres acteurs que les agriculteurs-éleveurs intervinrent dans le système paysager. Au tournant du XXe siècle, une voie de chemin de fer fut construite dans la haute vallée du Someş, facilitant l'exportation des ressources minérales et forestières. Une partie importante de la population active masculine travaillait dans les mines, dans des vallées méridionales (carrière de basalte depuis 1920 pour un quart des actifs), plus en amont dans la vallée du Someş (mines de zinc et de cuivre) ou dans la commune (carrière de marbre depuis 1930) ; déboisement de versants et bâtiments d'exploitation laissèrent des marques visibles dans le paysage des hauts du finage. La sylviculture et l'industrie du bois occupaient une autre partie des actifs; les versants forestiers se transformèrent alors lentement selon une politique cohérente impulsée par la direction sylvicole du département. Fondée en 1890, elle mit fin à l'économie de pillage ayant prévalu jusque-là ; l'administration roumaine prit le relais dans les années 1920 et établit des plans d'exploitation rationnelle des forêts (plantations d'épicéas) pour produire du bois d'oeuvre, destiné par exemple à la scierie de Maieru, propriété d'une famille juive de la commune. Du point de vue paysager, la dynamique entamée au XVIIIe siècle s'accentua : l'intervention de l'homme avait d'abord favorisé le hêtre, puis de plus en plus l'épicéa, au dépens d'autres espèces locales comme le sapin (Larion, 1982). Enfin, les potentialités touristiques de la région étaient déjà explorées (thermes jusqu'en 1914). En 1932, une première réserve naturelle fut créée dans la zone du Pietrosu $(2303 \mathrm{~m})$, juste à la limite nord du finage de Maieru (fig. 3). Le mécanisme décrit par Hirschhausen (2003) de "jeu complexe de fermeture/articulation par rapport au système englobant » des sociétés rurales montagnardes roumaines sous le régime communiste s'observe ici dès le début du siècle. En fait, parce que le "savoirmigrer " appartient à l'iconographie des populations, celle-ci put assurer à la fois l'efficacité du système agro-pastoral sur les paysages des bas et d'une partie des hauts et une articulation fructueuse du géosystème à un plus vaste ensemble, les effets de la circulation des productions (minerais, bois) s'intensifiant sur le paysage des hauts.

À l'époque communiste (fig. 4), le contrôle étatique du système paysager ne provoqua pas de rupture brutale. La superficie de l'ensemble des terres assurant le fonctionnement du système agro-pastoral fut réduite avec la confiscation des estives localisées hors du finage de la commune et la nationalisation des forêts privées et communales (1948), où une politique planifiée de régénération de la forêt fut menée par la régie nationale des forêts (Romsilva). Une scierie d'État fonctionnait à Maieru (le bois de hêtre était exporté vers les pays arabes). Un centre de collecte des petits fruits des bois fut ouvert sur le finage, riche en afinişuri (" champs de myrtilles »). L'élevage (16 000 têtes de bétail) passa sous le contrôle indirect de l'État qui imposa des quotas obligatoires de laine, de lait, de viande à livrer, mais l'absence de collectivisation des terres arables et des prés de fauche permit le maintien du parcellaire « en lames de parquet » (2,5 ha par famille). Le régime encouragea aussi la double activité : les cartes de planification prévoyaient entre 1972 et 1985 un triplement des «actifs de base en déplacement » de Maieru sur les sites d'exploitation minière. Toutefois, une partie des hommes prit l'habitude d'un travail saisonnier dans le bâtiment dans les villes du département et même à Cluj (fig. 1), durant leurs périodes de congé - indication d'un changement d'échelle du système englobant. Les activités touristiques se poursuivirent, mais restèrent marginales malgré l'extension des périmètres de réserves naturelles 
dans les Monts Rodna. Le système agro-pastoral put donc survivre et assurer l'entretien des paysages dont les dynamiques furent lentes, mais certaines. Elles sont liées à une pression démographique croissante, malgré l'exode des jeunes diplômés - Maieru ayant détenu pendant vingt-deux ans le record national de fécondité - et aux nouveautés techniques. En 1975, la route qui mène à Maieru fut asphaltée ; le pain confectionné à la maison fut concurrencé par celui des boulangeries industrielles. Sur les surfaces des bas $\mathrm{du}$ finage, les teintes jaunes disparurent de la palette de couleurs, car la culture des céréales fut progressivement abandonnée au profit des prés de fauche, confirmant la dynamique déjà engagée. Pommes de terre et légumes étaient encore produits, mais les habitants n'attendaient plus du géosystème leur autosubsistance. Les effets de la circulation furent aussi perceptibles sur le paysage de l'intravilan: les revenus complémentaires obtenus dans l'industrie furent investis dans l'habitat. Ces maisons plus vastes bâties à l'époque communiste ont des caractères reconnaissables : murs en briques ou en béton cellulaire, toits en bardeaux ou en zinc, étage (imposé par la réglementation) et balcon.

Ces dynamiques paysagères, passées souvent inaperçues, se confirment avec la dernière fluctuation de l'entre-deux (1989) (fig. 4). Les effets du «navettisme» prennent une ampleur nouvelle car les habitants de Maieru ont élargi l'horizon de leur savoir-migrer suite à la chute du régime communiste. Les fermetures d'usines (scierie) et de mines (Monts Rodna) et l'ouverture des frontières incitent la population active à dilater son territoire migratoire à l'espace européen. 20 \% travaillent toujours dans le bâtiment, surtout en Transylvanie (navettes hebdomadaires), mais $25 \%$ des actifs, activant ou réactivant des contacts ou des filières informelles (Églises, familles installées en Europe occidentale), sont partis comme saisonniers à l'étranger, surtout en Espagne, en Italie ou en Irlande. Leur objectif affiché est d'investir leur capital au village, où ils séjournent l'hiver; des entreprises de menuiserie ont ainsi été créées. Pourtant, le changement d'échelle de la circulation a fragilisé le système paysager: les prés de fauche, encore bien entretenus manuellement en bas de versant ont largement pris le pas sur des cultures réduites en fond de vallée à la production de pommes de terre, d'un peu de maïs et de légumes (photo. 3). La diminution du troupeau nuit à l'entretien des prés de fauche plus éloignés et des estives, et la reconquête de la forêt y est déjà visible. Les « anciens » interrogés sont aussi impressionnés par les changements de l'intravilan. Les rénovations et les constructions récentes de maisons réalisées grâce aux salaires gagnés à l'étranger ne passent pas inaperçues; malgré le maintien du balcon, les couleurs vives et la taille des nouveaux logements éclipsent le charme des dix dernières maisons en bois de sapin et la densification de l'espace bâti s'intensifie. Reste une impression générale d'harmonie et de permanence paysagères, mais le système actuel est désarticulé, car intégré dans un système englobant du type "mille-feuilles »- le territoire migratoire des stratégies individuelles, les effets de la filière bois sur l'étage forestier, le réseau planétaire des Réserves de la Biosphère - . En effet, en 2000, les réserves naturelles des Montagnes de Rodna ont changé de statut et d'échelle si bien que le finage de Maieru, comme celui de vingt-sept autres communes, se trouve aujourd'hui dans la zone-tampon et dans l'auréole de la zone de développement durable d'un Parc national de plus de 46000 ha. La future synthèse paysagère dépendra sans doute du succès des projets de cette Réserve de la Biosphère. On observe ici une forme de recommencement, car les anciennes communes des confins militaires appartiennent à la zone périphérique du parc; autrement dit, les descendants des 
populations mobilisées autrefois pour défendre les frontières de l'Empire sont maintenant sollicitées pour « défendre l'environnement ».

Photographie 3 : L'hôtel-château « Dracula » sur le Borgo pass (col de Tihuța, fig. 3), août 2008, C. Coumeff-Toader

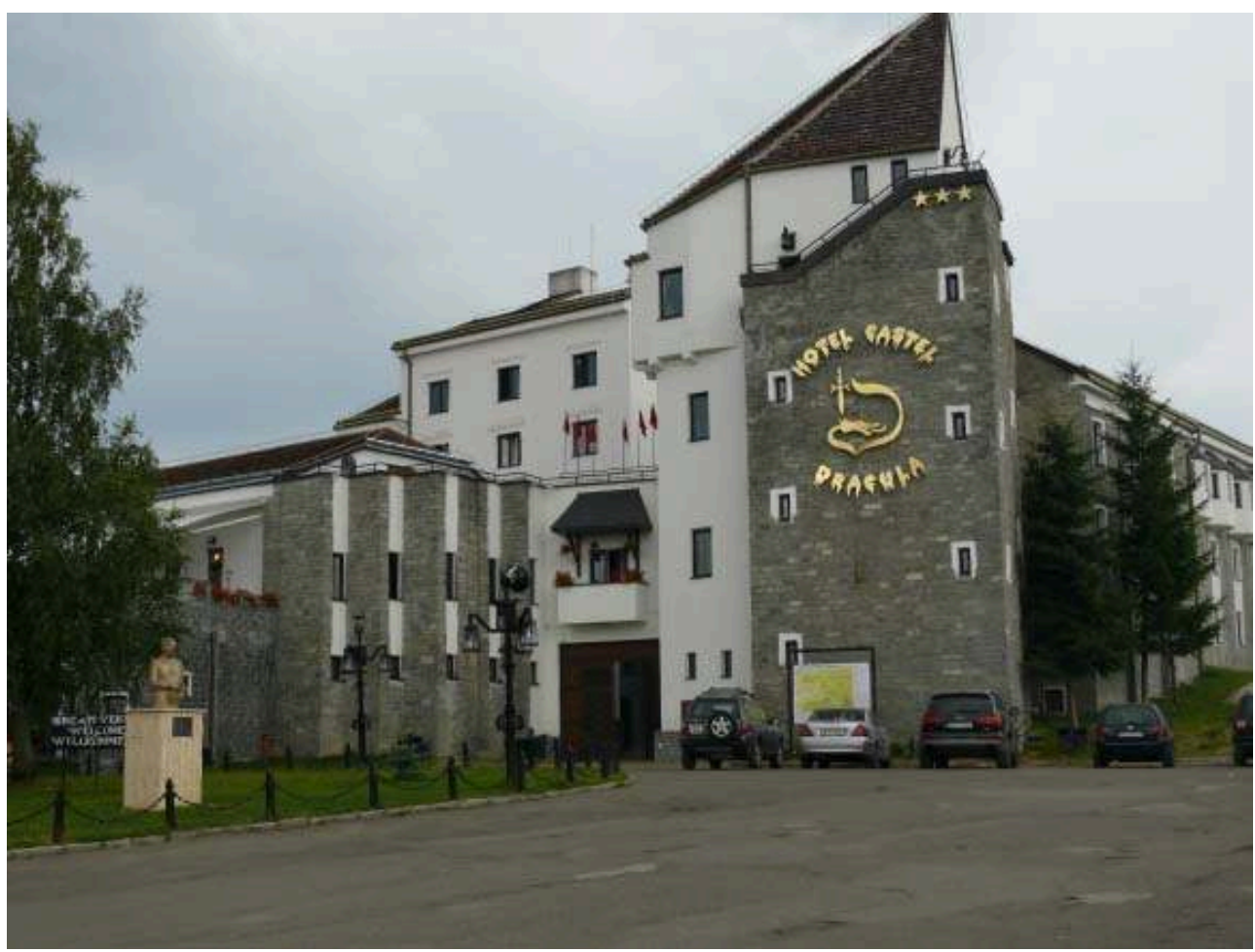

Bram Stoker s'inspira de la géographie des Carpates orientales pour situer le château de son personnage. Nul vestige médiéval n'existe sur le col, sur lequel a été construit un hôtel près d'une station de ski. 


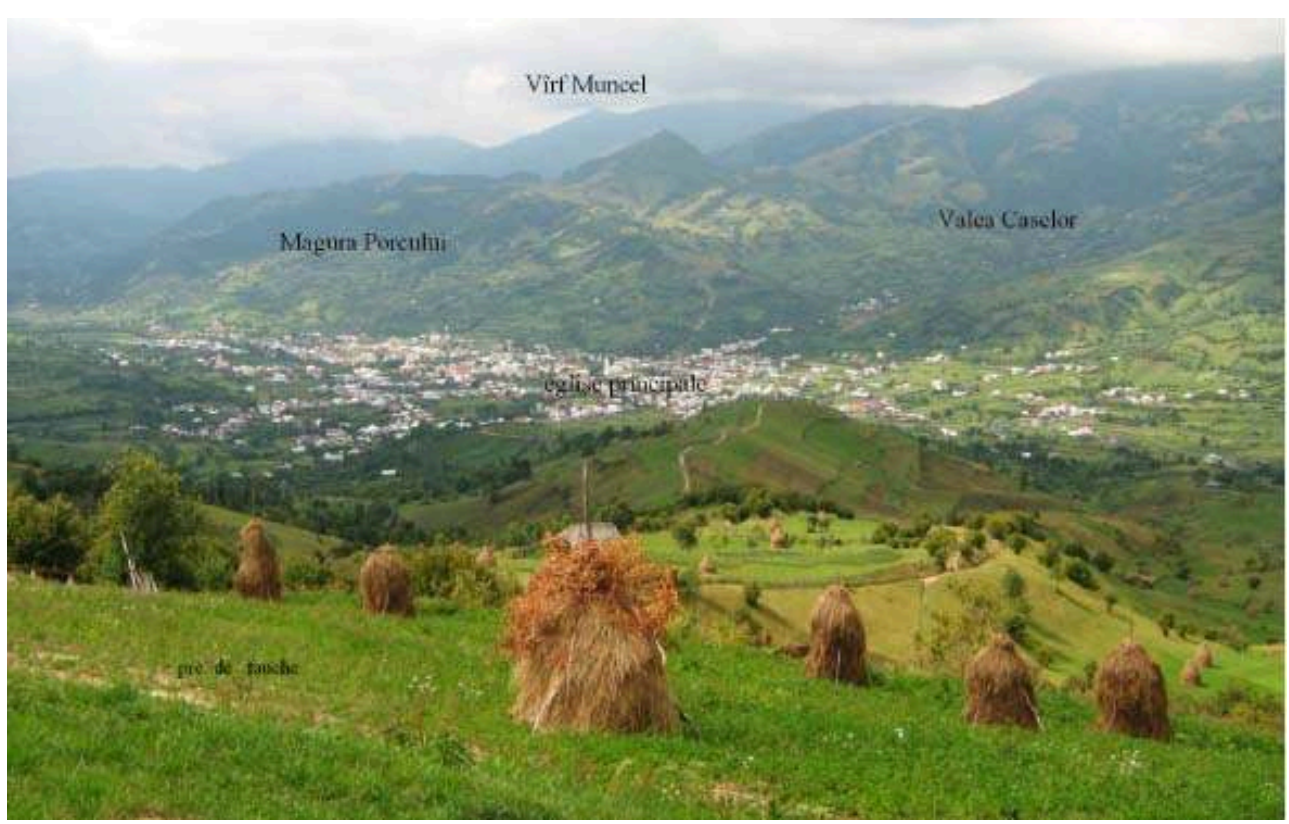

On repère les trois étages paysagers : le village et l'intravilan au fond de la dépression, cultures et prés sur les versants des collines, forêt au sommet des collines et sur les versants de la montagne, estives de haute altitude au dernier plan.

\section{3 - Cireşoaia, un système paysager de collines ou la véritable continuité}

Le site de la deuxième étude est localisé à la limite occidentale du département, sur le finage d'environ 900 ha de Cireşoaia (commune de Braniştea), un village perché à presque $600 \mathrm{~m}$ d'altitude dans une zone de collines qui domine au sud la vallée du Someş (fig. 3 et 5, photo. 4). L'impression de «bout du monde» notée par von Hirschhausen (1997) en 1991 reste la même en 2008, comme un écho au paysage de confins perdus des représentations hongroises. En effet, la population de ce village a la particularité d'être à $90 \%$ magyare et réformée. Le toponyme hongrois est MagyarDécse - du nom d'un cours d'eau sans doute - alors que le nom roumain est Cireşoaia mot dérivé de cireşe (cerises). C'est toute l'originalité et la force de l'iconographie locale, née d'une logique interne. Au XIXe siècle, les premières descriptions des occupations agricoles du finage signalent cultures de blé, vignes, prés de fauche et élevage, sur des versants encore largement boisés (Kadar, 1900). Un long et mauvais chemin desservait alors le village. Or, les habitants se transmettent une "légende " historiquement vraisemblable : au cours de la seconde moitié du XIXe siècle, un pasteur aurait demandé aux fidèles de planter un arbre fruitier avant leur mariage ou un baptême; cette initiative s'inscrivait dans un courant modernisateur de pasteurs et instituteurs réformés et unitariens, soucieux d'introduire de nouvelles méthodes pour améliorer les conditions de vie des paysans. Alliant le symbolisme biblique de l'arbre à celui de la fécondité familiale, prometteur d'enrichissement matériel, ce rite fit le succès de l'entreprise : au tournant du XXe siècle, une grande partie du finage était déjà couverte de vergers productifs et les villageois avaient initié une "fête des cerises " ouverte à tous et toujours célébrée. Ce mythe fondateur de la communauté accompagne 
un partage assez égalitaire des terres, favorisé par la réforme agraire de 1921. Une solidarité interne au village a instauré la gestion commune par quinze familles de la même rue de chacun des sept troupeaux d'ovins. La cohérence religieuse et sociale de cette iconographie simple a permis aux habitants de tirer parti de chaque fluctuation de l'entre-deux. L'analyse de ce géosystème circulaire à trois faciès paysagers dominants le montre.

L'Entre-deux-guerres fut la période de la conquête progressive du finage par l'arbre fruitier jusqu'à la constitution d'un système paysager abouti à la veille de la Seconde guerre mondiale. De petites maisons en bois et torchis, aux toits couverts de bardeaux occupaient alors l'intravilan; déjà les arbres fruitiers ombragaient rues et cours. Les vergers couvraient une auréole autour du village, puis gagnèrent les versants des vallons défrichés d'abord vers la plaine, puis jusqu'à la crête. Sur chaque parcelle étaient dispersés de façon aléatoire des arbres fruitiers de différentes espèces et variétés, l'habitude étant de planter les cerisiers au milieu et les pommiers sur les limites. Le verger fournissait du bois de chauffe après la taille et du foin après la fauche ; il était pâturé une partie de l'année par les ovins qui éliminaient les plantes adventices et fumaient le sol. À proximité du village, des parcelles de vigne, de cultures de blé, avoine et maïs, dont la production était destinée à la consommation familiale, et des parcelles boisées localisées dans le creux des vallons furent cependant préservées. Ce géosystème, dès cette époque, permettait à la population d'assurer son autosubsistance et de s'enrichir grâce à la vente des fruits. Celle-ci mit à son service la circulation: appel à la main-d'oeuvre extérieure pour la récolte, revente de la production dans les agglomérations de la vallée du Someş par de petites entreprises tenues par des familles juives. Les fruits partaient alors en charrettes tirées par des buffles ... Grâce à l'initiative des firmes commerciales et d'habitants du village émigrés aux États-Unis, de nouvelles techniques et variétés furent introduites. Le nombre de variétés de cerisiers est multiplié par sept de 1900 à 1945. Enfin, les modifications de frontières n'ont pas eu d'incidence majeure sur le fonctionnement régional du géosystème, les habitants surent même tirer parti de l'occupation hongroise (1940-1944) en faisant parvenir au parlement hongrois un panier de cerises et leurs doléances : un chemin direct et empierré reliant le village à la vallée fut construit (le chemin actuel). 
Fig. 5 : évolution du géosystème de Ciresoaia entre 1962 et le début du XXle siècle
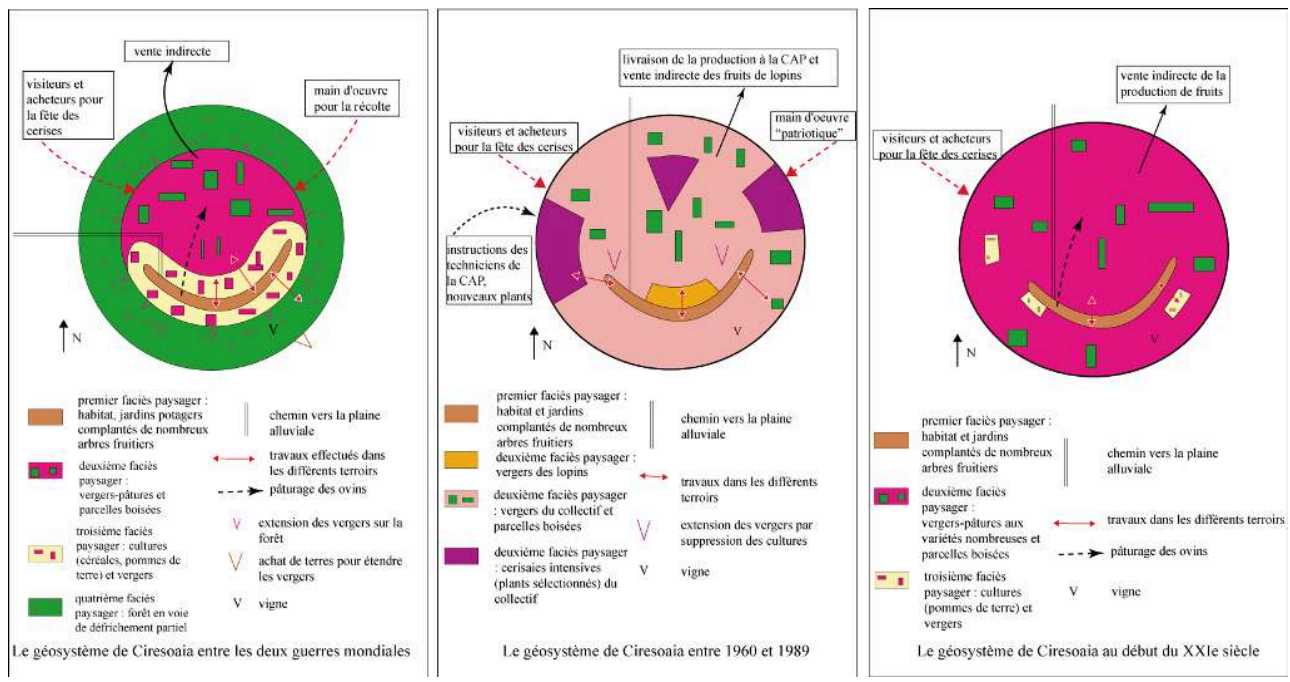

Pendant la période communiste, le système de quotas ne s'appliqua pas à la production de fruits. Jusqu'à la collectivisation (1960), le paysage de l'extravilan ne changea guère ; en revanche, les maisons en bois furent remplacées par des maisons en briques ou pierre, avec des couvertures en tuiles, financées par la vente des cerises. La collectivisation enleva aux villageois la maîtrise du système paysager qui s'inséra dans un système national rigide : la commercialisation de la récolte était assurée par l'État, les ingénieurs et techniciens venus de Moldavie modifièrent le parcellaire en plantant en alignements des plants de cerisiers sélectionnés sur une partie du finage. Mais la circulation ne bouleversa pas fondamentalement l'iconographie locale ni le faciès paysager du verger : la récolte était assurée par les lycéens dans le cadre du «travail patriotique ", le nombre de variétés de cerisiers doubla de 1960 à 1989, les surfaces consacrées aux vergers augmentèrent.

Von Hirschhausen (1997) témoigne de la rapidité avec laquelle les habitants ont dissout la CAP à la chute du régime communiste. La force de l'iconographie locale explique la capacité des villageois à orienter alors leur finage vers la spécialisation de l'arboriculture fruitière en associant techniques apprises à l'époque communiste (usage raisonné de traitements chimiques) et méthodes traditionnelles (mélange des espèces, suppression des cerisaies "intensives »). Quelques parcelles sont cultivées en maïs et pommes de terre. L'» étonnant désordre » (von Hirschhausen, 1997) du paysage ne doit pas faire illusion (photo. 5) : des petites entreprises de toute la Transylvanie viennent s'approvisionner en pommes et cerises à Cireşoaia pour les vendre sur les marchés urbains, et on rencontre sur le chemin les camions de quatre grossistes ukrainiens qui revendent la production jusqu'à Saint-Petersbourg. Une nouvelle synthèse paysagère est née, à l'intérieur d'un système englobant à l'échelle européenne. 


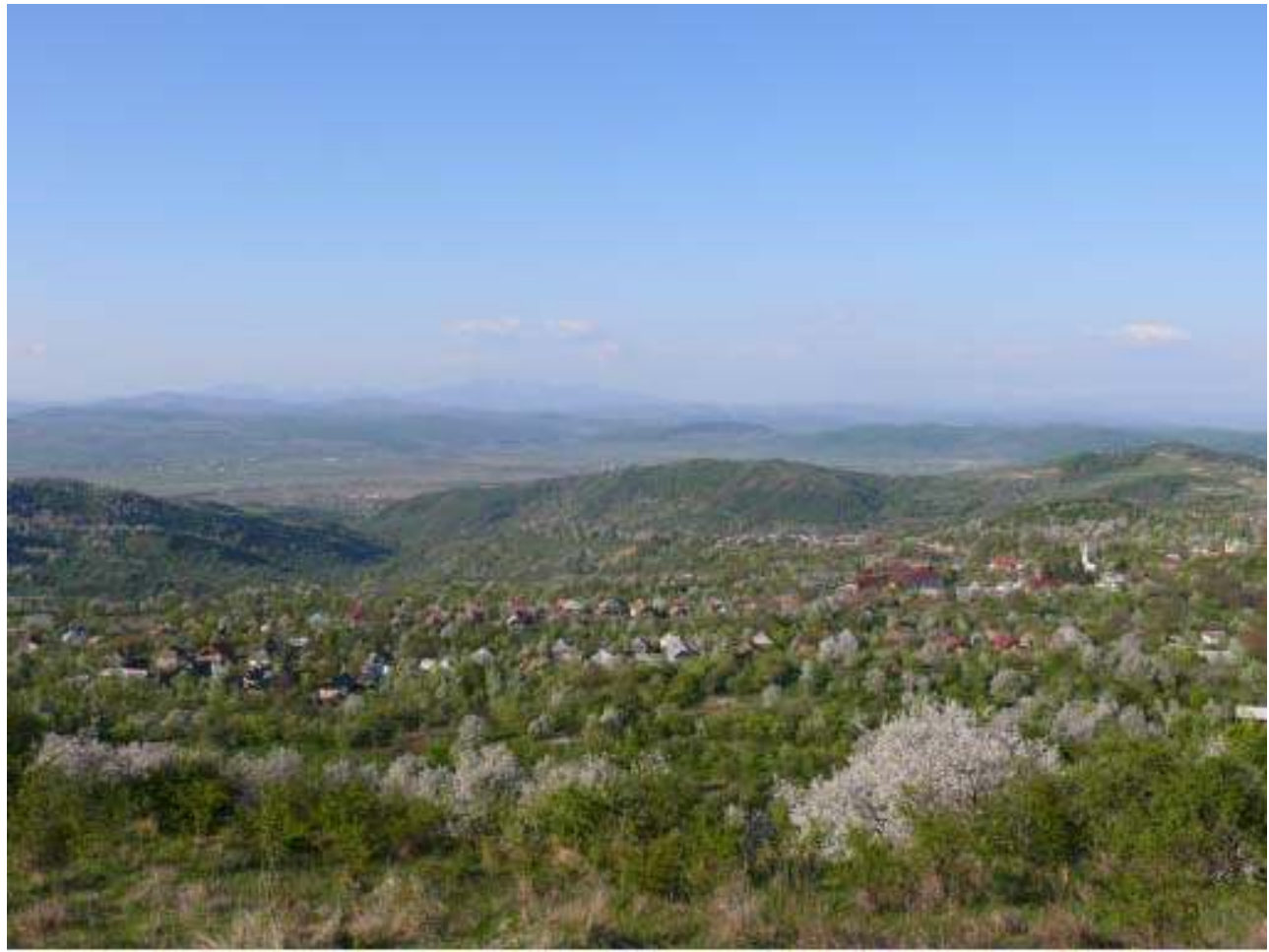

Le finage s'étend sur les versants des vallons couverts de vergers. C'est le moment de la floraison des cerisiers, repérables à leur couleur blanche. Le village s'étale sur la crête, on aperçoit le clocher du temple. Au dernier plan, on devine le fond de la vallée du Someş.

\section{4 - Florești, un système paysager de plaine ou l'impossible continuité}

Le dernier cas analysé est celui de Florești ou «village des fleurs » (fig. 3, 6, photo. 6), rattaché à la commune de Nimigea à partir des années 1950. Sa population est majoritairement roumaine (gréco-catholique, puis orthodoxe) depuis la fin du XVIIIe siècle. Le finage d'environ 700 hectares s'étend sur la rive gauche du Someş dans la plaine alluviale. La construction de l'iconographie locale s'achève dans les années 1920 et combine des données ambivalentes. Les habitants ont gardé la mémoire du second servage et de la lutte paysanne pour la propriété foncière. Les écoliers apprennent en cours d'Histoire qu'au XVe siècle, un des chefs d'une révolte de paysans de la vallée du Someş, durement réprimée, s'appelait Mihai Volah (Michel le Roumain) de Vireag (toponyme hongrois), c'est-à-dire de Floreşti, héros rural typique des mythes d'Europe centrale et orientale (Conte et Giordano, 1995). En effet, le servage puis un régime seigneurial lourd enchaînèrent les paysans du village jusqu'en 1863, année de la slobodna ou dezrobira: les villageois furent alors libérés des droits de leurs seigneurs magyars et une partie du finage fut partagée. Les stratégies d'acquisition de leur patrimoine foncier par les paysans conduisirent à un éparpillement et à un lanièrage de leurs parcelles, à un comportement malthusien dès la génération des couples nés vers 1900 et à une inégalité sociale entre petits et moyens propriétaires entre les deux guerres. À cette époque, les coutumes d'usage du finage prennent racine: le maïs devint la céréale centrale pour la consommation familiale et les cultures de légumes 
destinés à la vente (oignons au départ) firent l'originalité de Florești depuis que deux hommes originaires du village furent employés comme domestiques agricoles dans les fermes allemandes de la vallée de la Bistriţa (fig. 3) et transmirent la méthode de maraîchage intensif de plein champ qu'ils y avaient apprise. Les solidarités entre familles du village s'établissaient sur la base des alliances matrimoniales et du parrainage, mais aucune terre n'était communale et la gestion collective des troupeaux (bovins et ovins) respectait le principe de la propriété privée, de telle sorte que la solidarité villageoise était fragile. Le système paysager s'organise en quatre « quartiers».

Photographie 6 : Vue du finage de Floreşti vers le nord-ouest, avril 2009, C. Coumeff-Toader.

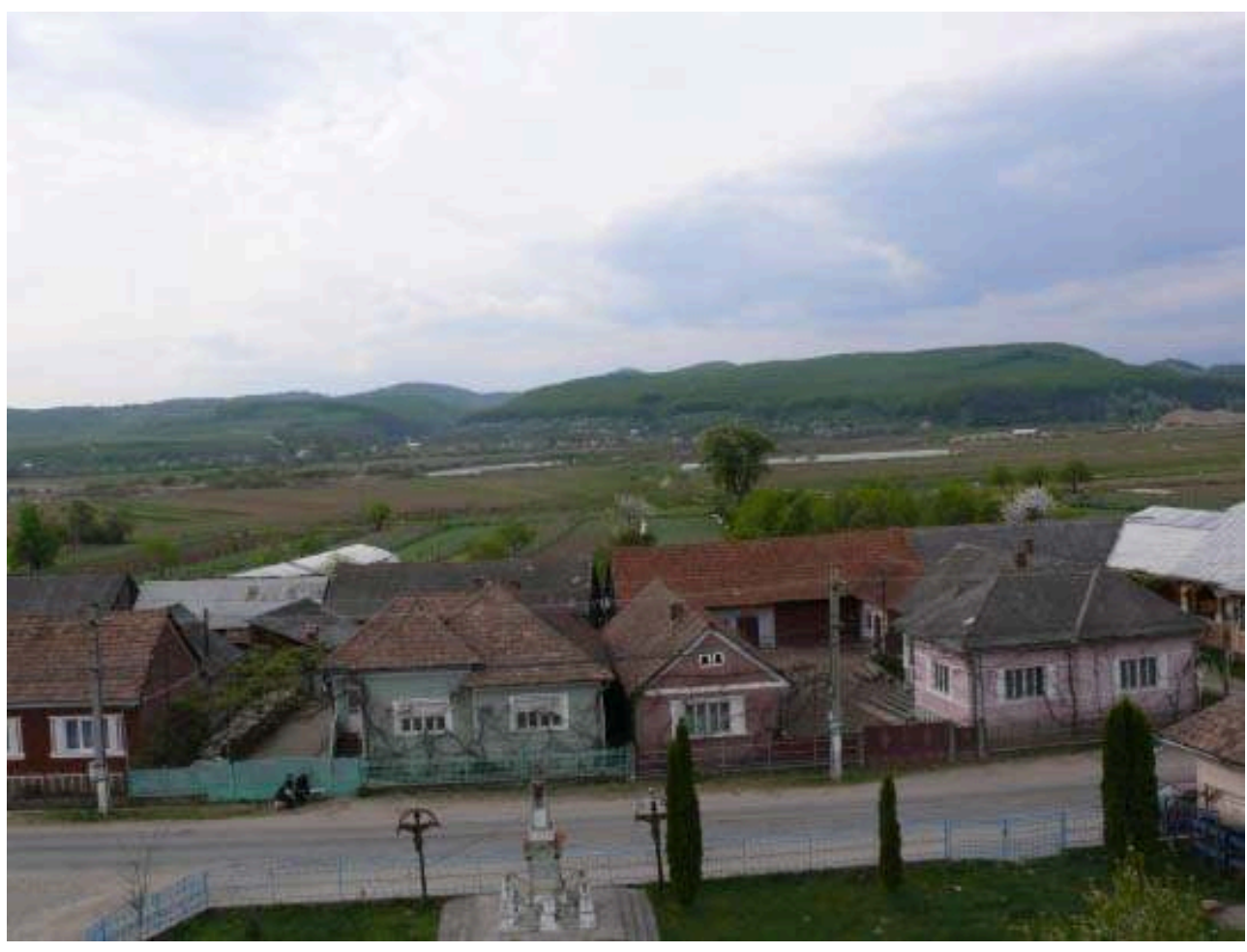

Le village occupe la rive orientale de la plaine alluviale du Someş. Au premier plan, le village et l' intravilan. On repère des serres dans les grands jardins derrière les maisons. Au second plan, les cultures maraîchères de plein champ sur les parcelles lanièrées. On devine le cours de la rivière, et au dernier plan, les sommets des monts Țibleş.

Au milieu des années 1930, le géosystème, dont les paysans étaient les seuls acteurs, était à son apogée, intégré dans un système englobant régional. L'intravilan offrait à la vue de petites maisons en bois et torchis non jointives, avec leur balcon et leur toit de chaume, et de grands jardins potagers. L'essentiel de la production de légumes (oignons, poivrons, cornichons, tomates) était réalisée en plein champ, sur les parcelles très étroites des sols légers près du Someş. Les sols marécageux au nord-est étaient consacrés aux prés de fauche. Les sols plus lourds au sud et à l'est voyaient se succéder en rotations complexes, gérées individuellement, céréales, pommes de terre, trèfle et lin, ou en association sur la même parcelle, maïs, courges, haricots et tournesol. Un effet paysager de tapis coloré en résultait. Les paysans devaient ponctuellement faire appel à la main-d'oeuvre des communes de montagne du Nord du département. Le maraîchage assura la réussite sociale à ces petits propriétaires qui allaient vendre en 
charrette leurs légumes sur les marchés urbains des vallées de la Bistrị̧a et du Someş, sachant mettre la circulation au service de leur iconographie.

L'irruption du régime communiste ne perturba pas dans un premier temps le géosystème, mais contribua à l'insérer dans un système englobant national. Compensant les pertes liées à la nationalisation de leurs forêts et au système des quotas, les paysans surent profiter du réseau ferré, aux tarifs abordables, pour vendre leurs légumes sur les grands marchés urbains du pays. Ils s'enrichirent et construisirent dans les années 1960 de plus grandes maisons en briques, avec un toit en zinc ou en tuiles. Mais en 1962 la collectivisation emprisonna les villageois dans un double système, celui du centre communal de la CAP à Nimigea, et celui de l'État central. La logique "productiste» (von Hirshhausen, 1999) du régime transforma le paysage de l'extravilan en un monotone parcellaire à larges mailles consacré aux céréales et pommes de terre; un projet d'aménagement des sols humides du quartier nord-est échoua. Les techniciens maintinrent au nord la culture de légumes en l'améliorant par l'irrigation par aspersion. S'arc-boutant sur leur iconographie, les habitants opérèrent un repli sur les jardins, - des lopins de 30 ares - : un maraîchage très intensif grâce au complantage d'espèces variées, en jouant sur leur durée différente d'arrivée à maturité, leur permettait d'obtenir plusieurs récoltes de carottes, persil-racine, radis, oignons et salades. L'impression paysagère de pêle-mêle répondait en fait à la logique de l'iconographie qui permettait de contourner celle du système communiste. De même, les paysans surent détourner à leur profit la circulation : leurs légumes frais en vente directe sur les marchés concurrençaient facilement ceux des magasins d'État et ils utilisèrent des réseaux informels afin de se procurer des pompes pour irriguer les jardins et de nouvelles semences pour enrichir leur palette de légumes.

Ce village connut une période de transition post-communiste difficile à cause de contestations sur les propriétés. Les paysans âgés s'efforcèrent de revenir au système agricole de leur jeunesse, mais ce "retour paysan» ne dura pas plus de dix ans. Aujourd'hui les trois-quarts des terres arables sont en friche, forêts et prés de fauche ne sont plus entretenus. Pourtant, dans les jardins de l'intravilan et dans le quartier nord, le maraîchage intensif se poursuit sous le mode d'un recommencement. Car une vingtaine de jeunes couples d'agriculteurs a décidé de spécialiser leur exploitation dans la « légumiculture ». Curieusement, sept d'entre-eux ont fait entre-temps le détour de l'immigration saisonnière (cueillette de cornichons) en Allemagne, profitant du réseau des Aussiedler (Michalon, 2003) et découvrant ainsi de nouvelles techniques. Ces agriculteurs dynamiques renouent de façon moderne avec l'esprit individualiste et capitaliste de l'Entre-deux-guerres: grandes serres (technique découverte à l'époque communiste), matériel moderne, nouvelles techniques d'irrigation, introduction de nouvelles espèces de légumes, fidélisation de la clientèle des villes du département, appel à la main-d'oeuvre rom locale ... Ils savent utiliser à leur profit la circulation à l'échelle départementale et européenne (semences). Par-delà la déchirure de l'époque communiste, une renaissance partielle et renouvelée du paysage de l'Entre-deuxguerres a donc émergé. 
Fig. 6 : évolution du géosystème de Floresti entre la période de l'Entre-deux-guerres et le début du XXIe siècle
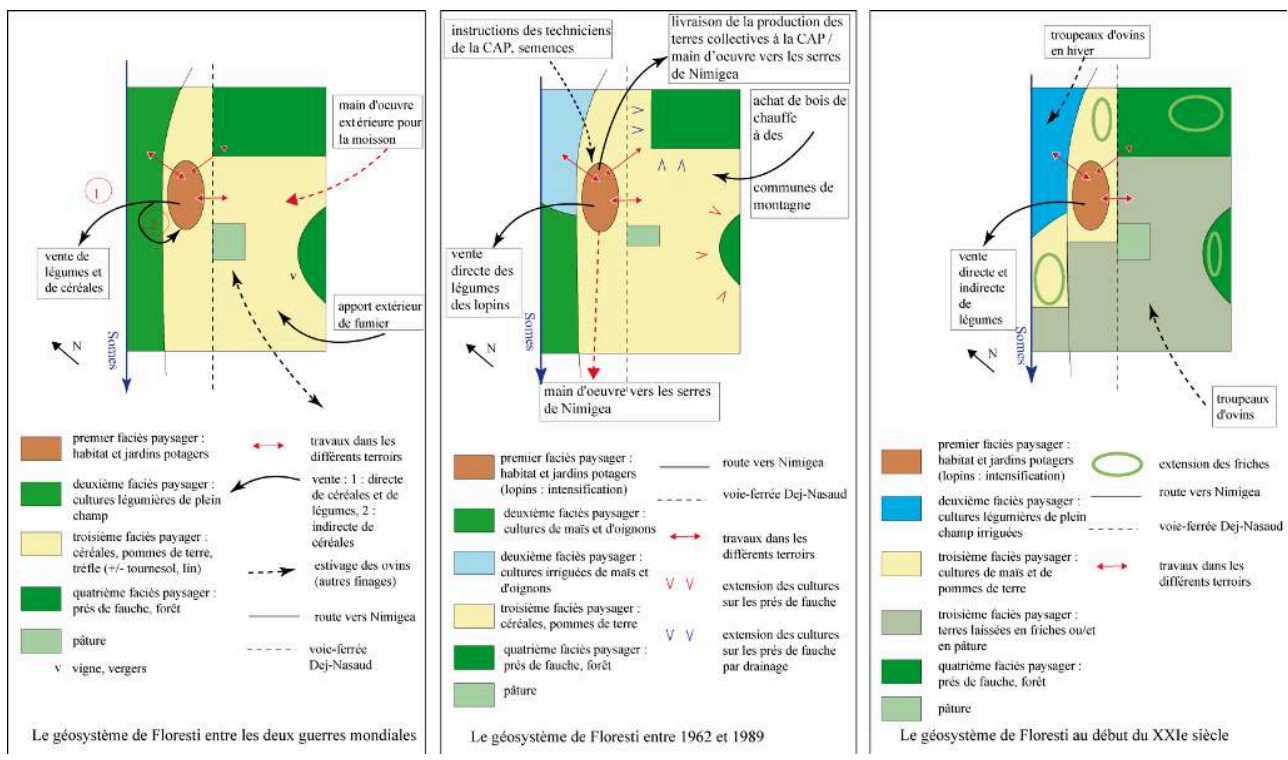

\section{Conclusion}

Dans les trois cas étudiés, la représentation d'un paysage immobile ne résiste pas à l'analyse géohistorique. Pourtant, les inflexions ou ruptures liées aux fluctuations de l'entre-deuxmontrent à chaque fois la capacité des sociétés locales, grâce à leur iconographie, à assurer l'adaptation ou la survie du système paysager, même si ce n'est pas toujours sur l'ensemble du finage. La question de la permanence du paysage n'est donc pas vaine : qu'est-ce qui lui confère ou lui dénie une continuité reconnaissable à travers le temps? Une réponse qui reconstruit les dynamiques paysagères tant sous leurs faces objective que subjective peut être avancée. Berque définit le paysage comme une des modalités de la "médiance ", la façon dont une société donne sens à son milieu, la relation qu'elle entretient avec son environnement. Par conséquent, le paysage est un rapport qu'on pourrait simplifier ainsi: Paysage $=$ Société $/$ Milieu . Le paysage de chaque société est issu d'une relation singulière au milieu, qui va lui donner un « style » reconnaissable, pour reprendre le vocabulaire de Blaga. Pour conserver une continuité paysagère, de telle sorte qu'on puisse écrire :

\begin{tabular}{|l|l|}
\hline Identité paysagère & = Société entre les deux guerres / Milieu entre les deux guerres \\
\hline & = Société de l'époque communiste / Milieu de l'époque communiste \\
\hline & $=$ Société actuelle / Milieu actuel \\
\hline
\end{tabular}

Ce rapport doit rester le même. Cela suppose un opérateur de rapports. Comme un coefficient de proportionalité, il assure l'égalité entre les générations de paysages. Cet opérateur ne peut être que l'iconographie puisque c'est la fonction des symboles de mettre en relation deux réalités (Ortigues, 2007). Chaque génération correspond grosso modo à une époque que les fluctuations de l'entre-deux délimitent nettement par des 
seuils. Si l'iconographie portée par une société joue cette fonction symbolique, alors les dynamiques paysagères s'inscrivent dans une continuité reconnaissable d'une génération de paysages à l'autre, tant du côté des représentations que des empreintes. Si la circulation a fortement bousculé les sociétés et leur iconographie, on constate que la continuité paysagère n'est maintenue que sur un espace réduit ou dans une représentation simplifiée, parfois même figée, en ce qui ne reste plus alors qu'un signe paysager.

Parmi les trois cas étudiés, celui de Cireşoaia illustre le mieux la capacité d'une société à garantir la continuité : au moment de la chute du régime communiste, le système paysager traverse un moment de crise et les habitants, sans en avoir vraiment conscience, sont revenus au seuil précédent de la généalogie pour qu'émerge une nouvelle synthèse paysagère mariant méthodes anciennes et nouvelles. La puissance de l'»opérateur", de l'iconographie, portée par une communauté forte, explique la brièveté de la crise. Pour comparer, à Florești, la même synthèse paysagère n'a pu s'opérer aujourd'hui que sur les deux quartiers du finage consacrés au maraîchage. Le repli sur l'espace domestique à l'époque communiste, la tradition jardinière et l'innovation réveillée par le détour saisonnier en Allemagne ont contribué à cette nouvelle configuration paysagère. À Maieru, la synthèse paysagère actuelle paraît fragile, encore sur le seuil. La spirale paysagère est rouverte avec la création de la Réserve de la Biosphère : quelles dynamiques paysagères doivent être encouragées au regard des représentations des populations urbaines occidentales et roumaines nostalgiques d'un paysage montagnard " authentique », dont les signes paysagers forts sont la meule de foin fauché à la main et la maison en bois avec son balcon? La deuxième composante de l'iconographie locale paraît aujourd'hui plus apte que la première à assurer la survie du système paysager, d'autant plus que la récente conjoncture économique menace les mouvements circulatoires des navettistes.

D'autres exemples d'analyses confirment l'ambivalence des signes paysagers, en fonction de leur degré de connexion au réseau de symboles transmis dans une société. L'approche généalogique permet de comprendre comment un système paysager peut finir par se figer dans un signe paysager isolé. Ainsi, la tradition paysagère saxonne, réduite au cliché du paysage "ethnique", est liée à une communauté qui a presque disparu. Pourtant, il reste des signes paysagers dans le département: les églises fortifiées. À Dumitra (fig. 3), la tour de guet (photo. 1) a été restaurée en 2003 grâce à l'aide financière des Aussiedler, les anciens habitants allemands encore attachés à leur iconographie et à la figure paysagère qui y est associée dans les confins perdus. Mais les villageois actuels n'assurent plus l'entretien global du système paysager. Le cas du paysage "fantastique » valide aussi cette approche généalogique, comme un contreexemple. Dans l'iconographie nationale roumaine, le surnom de Dracula renvoie à Vlad Tepeş, héros qui lutta contre l'invasion ottomane et maintint l'ordre en Valachie: aucune généalogie de paysages n'y est associée. Pourtant, à l'est du département de Bistri̧̧a-Năsăud, sur le col de Bărgău (pasul TihuŢa), là où l'imagination de Bram Stoker a placé le château du vampire, un signe paysagiste fantastico-médiéval a été construit de toutes pièces en 1983 à destination des touristes : un "hôtel-château " (photo. 3) très fréquenté par la clientèle américaine chaque 31 octobre, pour un rite annuel qui laisse ébahie la population locale. Ce signe paysager, totalement déconnecté de l'iconographie locale, a déjà fait entrer la Transylvanie dans une conception postmoderne (Berque, 1995) du paysage ... 
Les trois concepts testés lors de ces recherches - entre-deux, paysage-empreinte/ matrice, circulation/iconographie - ont montré leur pertinence dans l'étude des dynamiques paysagères. Pour sortir d'une conception figée et dissociée du paysage, souvent réduit d'un côté au palimpseste d'un support matériel ou de l'autre au regard et au vécu d'un sujet, la dialectique circulation/iconographie est féconde. Combinée à une approche généalogique, elle permet d'analyser de façon synthétique et dynamique les paysages et d'appréhender leurs évolutions selon une même logique, où la dimension symbolique et l'attention aux données physiques, historiques et sociales sont honorées. Pour le géographe, la Transylvanie est alors comme un laboratoire. Rey (1998) attribue aux espaces d'entre-deux les propriétés suivantes: la «confrontation entre forces externes et internes au dépens de ces dernières ", un " rapport au temps sur le mode du recommencement" et une "créativité élevée " mais "contrainte à s'évader ». Ces propriétés mettent en valeur les seuils des généalogies de paysages, révèlent comment les ruptures provoquent soit un recommencement, c'est-à-dire l'émergence d'une nouvelle synthèse paysagère, soit un épuisement du système paysager. Loin d'interpréter les réalités complexes des espaces d'entre-deux en termes de retard ou de décalage (Hirschhausen, Guest, 2008), cette étude montre qu'elles s'avèrent précieuses pour mettre à jour de façon démonstrative ce qu'on peut considérer comme le fonctionnement général de tout paysage: une généalogie à l'intérieur de laquelle se transmet un "style " paysager, une tradition vivante que chaque génération peut interpréter avec inventivité et raison, ou qui peut s'éteindre.

\section{BIBLIOGRAPHIE}

Bertrand C. et G. (2002) - Une géographie traversière : l'environnement à travers territoires et temporalités, Paris, Arguments, $331 \mathrm{p}$.

Berque A. (1995) - Les raisons du paysage, Paris, Hazan, 190 p.

Blaga L. (1991) - L'être historique, Paris, Librairie du Savoir, 243 p. En annexe : « L'éloge du village roumain » (1937).

Boia L. (2003) - La Roumanie. Un pays à la frontière de l'Europe, traduit du roumain par L. Rossion, Paris, Les Belles Lettres, $415 \mathrm{p}$.

Bruneau M. (2000) - « De l'icône à l'iconographie, du religieux au politique, réflexions sur l'origine byzantine d'un concept gottmannien ». Annales de Géographie, n 616, nov.-déc., p. 563-579.

Conte E. Giordano C. (1995) - « Sentiers de la ruralité perdue. Réflexions sur le postcommuniste », Études rurales, n 138-140, avril-déc. , p.11-33.

Coumeff-Toader C. (2008) - Représentations et dynamiques paysagères en Transylvanie de la fin du XIXe siècle à nos jours, le cas du département de BistriŢa-Năsăud, mémoire de Master 2 de géographie, Université de Nancy 2, 190 p. 
Dumas A. (1849) - « La dame pâle ». In : Les Mille et un Fantômes. Réédition séparée : Dumas A. (2006) - La dame pâle, Paris, Gallimard, 101 p.

Durandin C. (1995) - Histoire des Roumains. Paris, Fayard, 573 p.

Gusti D., Orghidan C., Vulcănescu M., Leonte V. (1938) - « JudeŢul Năsăud ». In : Enciclopedia României, vol. II, Bucureşti, Imprimeria NaŢională, pp. 298-303.

Hirschhausen B. von. (1997) - Les nouvelles campagnes roumaines. Paradoxes d'un « retour » paysan. Paris, Belin, 239 p.

Hirschhausen B. von, Patroescu M., Rey V. (1999) - « Les paysages agraires témoins de dilemmes de la transition roumaine ». In : Wicherek S. (dir.) Paysages agraires et environnement, principes écologiques de gestion en Europe et au Canada, Paris, CNRS Éditions, p. 61-72.

Hirschhausen B. von. (2003) - «La marginalité comme ressource : l'agriculture dans les Carpates roumaines face aux mutations nationales ». In : Crises et mutations des agricultures de montagne, Colloque international en hommage au professeur C. Mignon, Clermont-Ferrand, Presses Universitaires Blaise Pascal, p. 479-498.

Hirschhausen B. von, M. Guest. (2008) « Intégrer les campagnes bulgares et roumaines : le défi de leurs différences ». L'espace géographique, nº 4, p. 297-312.

Kadar J. (dir.) (1900) - Szolnok-Dobokavarmegye. Monographiaja, vol.II, Dej.

Larion C. (1982) - Flora şi aspecte de vegetatie din imprejurimili comunei Maieru, Jud.BistriŢa-Năsăud. ModalitaŢi de valorificate a cunostinelor de sistematica vegetala in lectiile de botanica şi filogenie vegetala. Lucrare pentru Gradul I didactic, Universitatea Babeş-Bolyai Cluj-Napoca, Facultatea de Biologie Geografie şi Geologie.

Michalon B. (2003) - « De la politique des Aussiedler à la circulation. Diversification des pratiques migratoires des Saxons de Transylvanie ». In : Diminescu D. (dir.) 2003, «Visibles, mais peu nombreux ». Les circulations migratoires roumaines. Paris, Éditions de la Maison des sciences de l'homme, p. 65-98.

Montaclair F. (1997) - Le vampire dans la littérature et au théâtre. Du mythe oriental au motif romantique. Besançon, Presses du Centre UNESCO, 263 p.

Mungiu-Pippidi A., Althabe G. (2004) - Villages roumains. Entre destruction communiste et violence libérale, Paris, L'Harmattan, 265 p.

Nouzille J. (1991) - Histoire des frontières : l'Autriche et l'Empire ottoman, collection Faits et représentations, Paris, Berg International, $263 \mathrm{p}$.

Nouzille J. (1993) - La Transylvanie, terre de contacts et de conflits. Strasbourg, Centre d'études germaniques, $255 \mathrm{p}$.

Oliveira C. de (1993) - L'impossibilité d'un autrement : la poésie allemande de Roumanie entre hétéronomie et dissidence (1944-1990). Thèse, Nancy II, 654 p.

Ortigues E. (2007) - Le discours et le symbole. Paris, Beauchesne, $261 \mathrm{p}$.

Prévélakis G. (2001) - «Circulation/iconographie contre Homme/Nature : Jean Gottmann et la délicatesse de la causalité ». In : Thumerelle P.-J. (dir.) Explications en géographie. Démarches, stratégies et modèles, Paris, SEDES, p. 41-55.

Rebreanu L. (1984, 3e éd.) - « Cuibul visurilor ». In : Rebreanu L., 1984, - Golani, nuvele si schite, Bucuresti, Editura Albatros, p. 251-259.

Rebreanu L. (1980) - 3e éd. Ion. Bucuresti, Editura Eminescu, 422 p. 
Rey V. (1998) - « Les territoires centre-européens, défis d'Europe ». In : Rey V. (dir.) 1998, Les territoires centre-européens. Dilemmes et défis. L'Europe médiane en question. Paris, La Découverte, $\mathrm{p}$. 13-33.

Rey V., Groza O., Ianos I., (dir.) (2000) - Atlas de la Roumanie. Collection Dynamiques du Territoire, Paris, CNRS-GDR Libergéo, La Documentation française, $167 \mathrm{p}$.

Rey V., Groza O. (2008) - « Bulgarie et Roumanie, un « entre-deux » géopolitique dans l'Union Européenne ». In : L’Espace géographique, $\mathrm{n}^{\circ} 4$, pp. 365-378.

Rougerie G., Beroutchachvili N. (1991) - Géosystèmes et paysages. Bilan et méthodes, col. U géographie, Paris, A. Colin, $302 \mathrm{p}$.

Stoker B. (1897) - Dracula, Archibald Constable and Company (première édition), 390 p.

Trégomain P. de (2005) - « Le syndrome du peuple élu : Les Saxons de Transylvanie et la destruction des confins ». Cultures $d^{\prime}$ Europe centrale, $n^{\circ}$ 5, CIRCE.

Verne J. (1892) - Le château des Carpathes, éd. P. -J. Hetzel.

Walter F. (2004) - Les figures paysagères de la nation : territoire et paysage en Europe (XVIe-XXe siècles). Paris, EHESS, $521 \mathrm{p}$.

\section{Site internet consulté}

Site du Parc national des Montagnes de Rodna. www.parcrodna.ro

\section{RÉSUMÉS}

Une approche géohistorique des paysages ruraux de Transylvanie à travers des études locales menées en 2006-2007 dans le département de BistriŢa-Năsăud montre l'intérêt des «espaces d'entre-deux» (Rey) pour analyser les dynamiques des paysages, abordés tant comme représentations que comme expressions des géosystèmes. Les évolutions des paysages de trois finages de la vallée du Someş, Maieru en moyenne montagne, Cireşoaia dans les collines, Floreşti dans la plaine alluviale, ont été reconstruites sur plus d'un siècle, de l'époque austro-hongroise au début du XXIe siècle, en passant par l'Entre-deux-guerres et la période communiste. L'enchaînement des systèmes paysagers, articulé à la succession des figures et imaginaires paysagers, a alors été interprété en termes de généalogies dont les seuils sont clairement mis en valeur par les fluctuations et ruptures politiques et culturelles. Pour expliciter le passage d'une génération de paysage à l'autre, il est nécessaire de prêter attention aux symboles (iconographie) portés par les sociétés locales. Ce modèle d'interprétation permet de rendre compte de l'identité d'un paysage, des continuités comme des ruptures paysagères.

A geohistoric approach of the rural landscapes of Transylvania through local studies carried out from 2006 to 2007 in the county of B-N shows the importance of the in-between spaces concept (Rey) to analyse the dynamics of the landscapes, studied as representations as well as expressions of geosystems. The landscapes of the three communal territories of the Somes valley (Maieru situated in medium-sized mountains, Ciresoaia in the hills, Floresti in the alluvial plain) kept being remodelled for more than a century, from the Austro-Hungarian period to the beginning of the 21st century, including the time between the two World Wars and the Communist period. The logical sequence of the landscape systems, in relation to the the succession of figurative and imaginary landscapes, was then interpreted in terms of genealogy, the thresholds of which are clearly highlighted by the fluctuations and disruptions in the fields of politics and culture. To 
clarify the passage from one generation of landscape to another, it is necessary to pay attention to the symbols (iconography) to be found in local societies. This model of interpretation enables us to show the identity of a landscape, as well as any continuities and discontinuities.

\section{INDEX}

Keywords : Transylvania, landscape, between spaces, geosystem, geohistory, circulation/ iconography

Mots-clés : Transylvanie, paysage, espace d'entre-deux, géosystème, géohistoire, circulation/ iconographie

\section{AUTEURS}

\section{DOMINIQUE HARMAND}

Centre d'Etudes et de Recherches sur les Paysages (CERPA). Campus Lettres Sciences Humaines. BP 1339754015 NANCY CEDEX 